\title{
Influência da ação antrópica sobre a fauna de macroinvertebrados aquáticos em riachos de uma região de cerrado do sudoeste do Estado de São Paulo
}

\author{
Sara S. Cortezzi ${ }^{1,2}$, Pitágoras da C. Bispo ${ }^{1}$, Gabriel de P. Paciencia ${ }^{1,3}$ \& Ricardo C. Leite $^{1,3}$ \\ 1. Laboratório de Biologia Aquática, Departamento de Ciências Biológicas, Faculdade de Ciências e Letras de Assis, Universidade Estadual \\ Paulista. Av. Dom Antônio, 2100, Parque Universitário, 19806-900 Assis, SP, Brasil. (saracortezzi@yahoo.com.br; \\ pitágoras@assis.unesp.br; gabrielpaciencia@yahoo.com.br; ricardocardosoleite@yahoo.com.br) \\ 2. PPG em Zoologia, Departamento de Zoologia, Instituto de Biociências de Botucatu, Universidade Estadual Paulista, Botucatu, SP, \\ Brasil. \\ 3. PPG em Entomologia, Departamento de Biologia, Faculdade de Filosofia, Ciências e Letras de Ribeirão Preto, Universidade de São \\ Paulo, Ribeirão Preto, SP, Brasil.
}

\begin{abstract}
Anthropic action influence on aquatic macroinvertebrates in streams from a cerrado region of Southwestern of the State of São Paulo, Brazil. The richness and spatial distribution of aquatic macroinvertebrates were studied in streams from a cerrado region, Assis, State of São Paulo, with the purpose of verifying the effect of the anthropic action on the fauna. The collections took place at the headwaters of the Ribeirão Água do Cervo (the main water supplier of the city of Assis). Nine collection stations were established in which ten standardized pebble packages were placed (equivalent volume, $300 \mathrm{~cm}^{3}$ each). After twenty-five days of exposure, the packages were removed from the stream. The macroinvertebrates associated to each of the pebble packages were removed and identified. The results showed a smaller richness in the most damaged station. There was not significant relation between the physicochemical factors and the fauna. The intensity of anthropic action was significantly related with the fauna.
\end{abstract}

KEYWORDS. Organic pollution, aquatic insects, lotic ecosystems.

RESUMO. Foram estudadas a riqueza e a distribuição espacial da fauna de macroinvertebrados aquáticos em riachos da região de cerrado de Assis, SP, com a finalidade de verificar o efeito da ação antrópica sobre a fauna. As coletas foram realizadas em nascentes do Ribeirão Água do Cervo, principal fornecedor de água da cidade de Assis. Foram estabelecidos nove pontos de coleta nos quais foram colocados dez pacotes de seixos de tamanho padronizado (volume equivalente a $300 \mathrm{~cm}^{3}$ cada). Após 25 dias de exposição, os pacotes foram removidos do riacho. Os macroinvertebrados associados a cada pacote de seixos foram triados e identificados. Os resultados mostraram uma menor riqueza no local mais impactado. Não houve relação significativa entre os fatores físico-químicos e a fauna. No entanto, a ação antrópica foi significativamente relacionada com a fauna.

PALAVRAS-CHAVE. Poluição orgânica, insetos aquáticos, ecossistemas lóticos.

A riqueza, a densidade e a composição de macroinvertebrados aquáticos variam no tempo e no espaço como resultado da influência de fatores ambientais e bióticos (VANNOTE et al., 1980; DinIZ-FILHO et al., 1998; BAPTisTA et al., 2001; BisPo et al., 2006). Neste sentido, o tamanho do riacho, as fontes predominantes de entradas de energia (alóctone e/ou autóctone), a frequência e a intensidade de perturbações, a heterogeneidade ambiental e as interações bióticas (VANNOTE et al., 1980; Hildrew \& Towsend, 1987; McCabe \& Gotelli, 2000; Voeltz \& McArthur, 2000) podem ser alguns dos importantes fatores determinantes da diversidade de macroinvertebrados aquáticos em ambientes lóticos. Aliado a isso, atualmente, as atividades humanas têm sido responsáveis pela redução da riqueza e pela modificação da composição faunística de macroinvertebrados aquáticos em ambientes lóticos (Rosenberg \& Resh, 1993; Zamora-Muñoz \& AlbATercedor, 1996; ShIEH et at., 1999; MoORE \& PALMER, 2005).

Os macroinvertebrados aquáticos possuem uma grande diversidade ecológica, sendo que muitas espécies são altamente sensíveis às perturbações ambientais, enquanto outras são tolerantes aos diferentes impactos (Rosenberg \& Resh, 1993). Adicionalmente, estes organismos apresentam baixa mobilidade, sofrendo portanto, os efeitos dos impactos antrópicos locais. As modificações estruturais do habitat e a liberação de efluentes (diminuindo a qualidade da água) são fatores importantes e afetam diretamente a fauna de macroinvertebrados aquáticos. Por isto, estes organismos têm sido frequentemente utilizados na avaliação e biomonitoramento da qualidade ambiental de ecossistemas aquáticos (BARBOUR et al., 1996; BAPTISTA et al., 2007).

Neste contexto, o presente trabalho teve como objetivo estudar a riqueza e a distribuição espacial da fauna de macroinvertebrados aquáticos em riachos da região de cerrado de Assis, São Paulo, com o intuito de verificar o efeito da ação antrópica sobre a fauna.

\section{MATERIAL E MÉTODOS}

Área de estudo. O trabalho foi realizado em riachos do município de Assis, província Geomorfológica do Planalto Ocidental, sudoeste do Estado de São Paulo. Foram estudadas as nascentes do Ribeirão Água do Cervo. Estas nascentes compreendem riachos que nascem em área protegida (Estação Ecológica e Floresta Estadual de Assis), em áreas agrícolas e em área urbana. O Ribeirão Água do Cervo é o principal fornecedor de água para o município de Assis. A altitude varia entre 500 e 588 m. A 
vegetação original é composta de cerradão (sistema florestal pertencente ao domínio morfoclimático do cerrado, no qual há um predomínio do componente arbóreo e arbustivo). Remanescentes dessa vegetação ainda estão preservados na unidade de conservação. Ao longo dos trechos de alguns riachos persistem matas ciliares.

Coletas. Foram utilizados seixos para padronizar a coleta de dados. Estes foram distribuídos em pacotes de tamanho padronizado, com volume equivalente a $300 \mathrm{~cm}^{3}$ cada. Dez pacotes foram colocados aleatoriamente no leito de nove pontos de coleta onde permaneceram por 25 dias. As datas das retiradas foram 12/XII/05 para os pontos 1 e 2, 13/XII/05 para o ponto 3 e 16/XII/05 para os pontos $4,5,6,7,8$ e 9 . Com o objetivo de evitar a perda de indivíduos, as retiradas foram feitas com o auxílio de uma rede de coleta $(0,25 \mathrm{~mm}$ de malha). Os seixos foram lavados e os organismos separados e acondicionados em álcool 80\%. Posteriormente, estes foram triados e identificados.

Caracterização Ambiental. Cada trecho amostrado foi avaliado quanto à cobertura vegetal, qualidade estrutural do habitat, presença de atividade agrícola, proximidade de residências e despejo de efluentes. Estas informações foram utilizadas para ordenar os pontos em uma escala de cobertura vegetal e intensidade de ação antrópica. A cobertura vegetal foi determinada dentro de uma escala de zero a três $(0$, nenhuma; 1 , baixa; 2 , média; 3 , alta) e a intensidade de ação antrópica de zero a quatro (no caso desse fator, foi utilizada a mesma escala acima, incluindo o nível 4, para encaixar um dos pontos que apresentou ação antrópica muito superior aos demais). Em cada um dos pontos amostrados, também foram registrados os seguintes fatores ambientais: temperatura da água $\left({ }^{\circ} \mathrm{C}\right)$, velocidade $(\mathrm{m} / \mathrm{s})$, vazão $\left(\mathrm{m}^{3} / \mathrm{s}\right)$, potencial hidrogeniônico $(\mathrm{pH})$, condutividade elétrica $\left(\mu \mathrm{S} . \mathrm{cm}^{-1}\right)$, oxigênio dissolvido (mg. $\left.1^{-1}\right)$ e turbidez (NTU). A velocidade foi obtida através de um fluxômetro. A vazão foi calculada como o produto da largura, pelas médias da velocidade e da profundidade. Os demais fatores foram registrados através de equipamento para análise de água. A caracterização dos pontos coletados é apresentada na tabela I.

Identificação taxonômica. Oligochaeta e Hirudinea foram mantidos em grandes grupos e os demais macroinvertebrados foram separados em morfoespécies. No caso de Chironomidae (Diptera), adotamos um critério mais conservador, dessa forma consideramos apenas uma morfoespécie para cada gênero.
Análise de dados. O número de indivíduos coletados variou muito entre os pontos, portanto, os valores de riqueza obtidos podem ter um viés. Para resolver este problema, a riqueza foi avaliada através de curvas de rarefação (Hulbert, 1971; Simberloff, 1972) usando aleatorização. Para estimar o número de táxons para $\mathrm{k}$ indivíduos, $\mathrm{k}$ indivíduos foram retirados aleatoriamente da amostra e o número observado de táxons foi registrado. Esse procedimento foi repetido mil vezes usando o programa Ecosim 5 (Gotelli \& ENTSMINGER, 2000).

A estrutura da comunidade foi avaliada por métodos multivariados (MANLY, 1994). A matriz de dados foi logaritmizada e posteriormente submetida à Análise de Correspondência Destendenciada, DCA (GAUCH JR., 1995; JonGMAN et al., 1995; LEGENDRE \& LEGENDRE, 1998). A matriz original também foi submetida ao método de classificação divisiva TWINSPAN ("Two Way Indicator Species Analysis"), considerando os seguintes níveis de corte para estabelecer as diferentes pseudoespécies: zero, 30, 60, 100 e 200 indivíduos (GAUCH JR., 1995). Para testar o efeito dos fatores físico-químicos, da distância geográfica e da ação antrópica sobre a fauna foi utilizado o teste de Mantel com 5.000 permutações (MANLY, 1994; LEGENDRE \& LEGENDRE, 1998). No caso da fauna, foi utilizado o Índice de Morisita-Horn (HoRn, 1966; WoldA, 1981; KREBS, 1999) como critério para estabelecer a similaridade faunística entre os pontos. No caso dos fatores físico-químicos, uma matriz de distâncias euclidianas entre os pontos foi obtida a partir da matriz logaritmizada e padronizada. As análises foram realizadas utilizando os programas PC-ORD 4.0 (McCunE \& MEFFORD, 1999) e NTSYS 2.1 (Rohlf, 2000). Parte dos organismos jovens não foi considerada na análise devido à impossibilidade de separá-los nas diferentes morfoespécies. Para as famílias de Coleoptera representadas tanto por larvas quanto por adultos, apenas as primeiras foram incluídas na análise, devido à impossibilidade de relacionar os adultos e as larvas.

\section{RESULTADOS}

Foram coletados 3.924 indivíduos distribuídos em 96 táxons. A lista dos invertebrados coletados, com os respectivos pontos de coleta, é apresentada na tabela II. As curvas de rarefação para a fauna de

Tabela I. Fatores Ambientais registrados em nove pontos de coleta em riachos da região de Assis, Estado de São Paulo. Potencial hidrogeniônico $(\mathrm{pH})$, condutividade elétrica $\left(\mathrm{CE}, \mu \mathrm{S} . \mathrm{cm}^{-1}\right)$, vazão $\left(\mathrm{m}^{3} / \mathrm{s}\right)$, velocidade da água (Vel., m/s), turbidez (Turb., NTU), oxigênio dissolvido (OD, mg. $1^{-1}$ ), temperatura da água (Temp., ${ }^{\circ} \mathrm{C}$ ), intensidade de cobertura vegetal (CV, escala de 0 a 3 ) e intensidade de ação antrópica (AA, escala de 0 a 4; 0, nenhuma; 1, baixa; 2, média; 3, alta; 4, extremamente alta).

\begin{tabular}{|c|c|c|c|c|c|c|c|c|c|}
\hline Pontos & 1 & 2 & 3 & 4 & 5 & 6 & 7 & 8 & 9 \\
\hline Coord. S & $22^{\circ} 35^{\prime} 22^{\prime \prime}$ & $22^{\circ} 35^{\prime} 33^{\prime \prime}$ & $22^{\circ} 36^{\prime} 16^{\prime \prime}$ & $22^{\circ} 36^{\prime} 26^{\prime \prime}$ & $22^{\circ} 36^{\prime} 26^{\prime \prime}$ & $22^{\circ} 37^{\prime} 00^{\prime \prime}$ & $22^{\circ} 37^{\prime} 01^{\prime \prime}$ & $22^{\circ} 36^{\prime} 50^{\prime \prime}$ & $22^{\circ} 38^{\circ} 26^{\prime}$ \\
\hline Coord. W & $50^{\circ} 24^{\prime} 44^{\prime \prime}$ & $50^{\circ} 25^{\prime} 23^{\prime \prime}$ & $50^{\circ} 25^{\prime} 44^{\prime \prime}$ & $50^{\circ} 25^{\prime} 52^{\prime \prime}$ & $50^{\circ} 25^{\prime} 52^{\prime \prime}$ & $50^{\circ} 25^{\prime} 16^{\prime \prime}$ & $50^{\circ} 24^{\prime} 36^{\prime \prime}$ & $50^{\circ} 24^{\prime} 59^{\prime \prime}$ & $50^{\circ} 25^{\prime} 08^{\prime}$ \\
\hline $\mathrm{pH}$ & 7,07 & 8,80 & 6,60 & 7,00 & 7,90 & 7,90 & 7,90 & 6,85 & 5,90 \\
\hline $\mathrm{CE}$ & 10 & 3 & 4 & 40 & 19 & 25 & 2 & 3 & 81 \\
\hline Vazão & 0,020 & 0,038 & 0,059 & 0,026 & 0,276 & 0,099 & 0,051 & 0,097 & 0,126 \\
\hline Vel. & 0,517 & 0,311 & 0,232 & 0,242 & 0,706 & 0,298 & 0,131 & 0,598 & 0,948 \\
\hline Turb. & 5,0 & 1,0 & 0 & 0 & 5,0 & 8,5 & 1,0 & 0 & 3,0 \\
\hline OD & 5,25 & 7,80 & 6,50 & 7,14 & 7,15 & 6,88 & 6,55 & 7,66 & 3,63 \\
\hline Temp. & 22,6 & 27,3 & 22,0 & 22,5 & 22,1 & 24,0 & 22,0 & 24,4 & 31,3 \\
\hline $\mathrm{CV}$ & 2 & 0 & 3 & 2 & 2 & 0 & 3 & 1 & 0 \\
\hline $\mathrm{AA}$ & 0 & 3 & 1 & 1 & 2 & 3 & 0 & 0 & 4 \\
\hline
\end{tabular}


macroinvertebrados aquáticos nos nove pontos de coleta são apresentadas nas figuras 1-5, a riqueza foi padronizada para 150 indivíduos (Fig. 6). No presente trabalho, a riqueza foi menor no ponto 9 , local extremamente impactado pela ação antrópica (intensidade 4) (Fig. 6). O ponto 6 (intensidade de ação antrópica 3), foi o segundo ponto com menor riqueza. $\mathrm{O}$ ponto 7 , local com nenhuma ação antrópica (intensidade zero), foi o que apresentou maior riqueza. Quanto aos demais pontos, as riquezas dos pontos 1 e 3 (intensidade de ação antrópica zero e 1) se sobrepuseram levemente ao intervalo de confiança (95\%) do ponto 2 (intensidade 3); aliado a isso, a riqueza do ponto 4 (intensidade de ação antrópica 1) se sobrepôs ao intervalo de confiança do ponto 5 (intensidade de ação antrópica 3) (Tab. I, Fig. 6). Estes resultados indicam que alguns pontos podem ter riquezas similares, apesar de estarem submetidos a diferentes intensidades de ação antrópica. $\mathrm{O}$ ponto 8 não foi incluído na Fig. 6 pelo fato de apenas 69 indivíduos terem sido coletados neste local e a padronização ter sido feita para um número mais representativo, 150 indivíduos.

O primeiro eixo da DCA explicou $61,4 \%$ da variabilidade e separou o ponto 9 dos demais pontos de

Tabela II. Macroinvertebrados aquáticos em nove pontos de coleta em riachos da região de Assis, Estado de São Paulo (A, adulto; L, larva).

\begin{tabular}{|c|c|c|c|c|c|}
\hline Táxons & $\begin{array}{l}\text { Pontos de } \\
\text { ocorrência }\end{array}$ & Táxons & $\begin{array}{l}\text { Pontos de } \\
\text { ocorrência }\end{array}$ & Táxons & $\begin{array}{l}\text { Pontos de } \\
\text { ocorrência }\end{array}$ \\
\hline ARTHROPODA & & Coleoptera L sp.1 & 1 & Chironomidae & \\
\hline Collembola & & Coleoptera L sp.2 & 6 & Ablabesmya sp. & $2,3,4,8$ \\
\hline Collembola sp.1 & 9 & Coleoptera L sp.3 & 6 & Beardius sp. & $1,2,3,4,7,8$ \\
\hline Insecta & & Coleoptera L sp.4 & 7 & Chironomus sp. & 7,9 \\
\hline Ephemeroptera & & Coleoptera A sp.1 & 2 & Cladotanytarsus sp. & $1,3,4$ \\
\hline Leptophlebiidae & & Coleoptera A sp.2 & 5 & Corynoneura sp. & $1,2,4,5,7$ \\
\hline Hagenulopsis sp.1 & 1 & Coleoptera A sp.3 & 5 & Cricotopus sp. & $1,2,3,4,5,7,8$ \\
\hline Miroculis sp.1 & 1 & Coleoptera A sp.4 & 6 & Djaumabatista sp. & 7,9 \\
\hline Baetidae & & Coleoptera A sp.5 & 7 & Fissimenteum sp. & $2,4,6,7,8$ \\
\hline Tupiara sp.1 & 6 & Trichoptera & & Kiefferulus sp. & 7 \\
\hline Caenidae & & Hydropsychidae & & Labrundinia sp. & 3 \\
\hline aff. Brasilocaenis sp.1 & 3 & Macronema sp.1 & 6 & Lopescladius sp. & $3,4,7$ \\
\hline Odonata & & Leptonema sp.1 & $1,2,3$ & Manoa sp. & 2,6 \\
\hline Gomphidae sp.1 & $1,2,3,4$ & Leptonema sp.2 & $2,5,6$ & Nanocladius sp. & $3,4,7,8$ \\
\hline Gomphidae sp.2 & 3 & Leptonema sp.3 & $2,4,5$ & Parachironomus sp. & 3 \\
\hline Libellulidae sp.1 & 1,7 & Leptonema jovens & $1,2,3,4,5,6,7,8$ & Parametriocnemus sp. & $3,4,7$ \\
\hline Libellulidae sp.2 & $1,2,3,4,5,6,7,8$ & Smicridea sp.1 & $2,6,8$ & Pentaneura sp. & $1,2,3,4,5,6,7,8$ \\
\hline Libellulidae sp.3 & $2,5,6,8$ & Smicridea $\mathrm{sp} .2$ & 2 & Phaenopsectra sp. & 1 \\
\hline Libellulidae sp.4 & $2,3,6$ & Smicridea jovens & 2 & Polypedilum spp. & $1,2,3,4,5,6,7$ \\
\hline Libellulidae sp.5 & 2 & Blepharopus sp.1 & $4,5,7,8$ & Procladius sp. & 3 \\
\hline Calopterygidae sp.1 & 1,2 & Calamoceratidae & & Pseudochironomus sp. & 2,5 \\
\hline Calopterygidae sp.2 & 3 & Phylloicus sp.1 & 7 & Rheotanytarsus sp. & $1,2,3,4,5,6,8,9$ \\
\hline Plecoptera & & Odontoceridae & & Saetheria sp. & $1,2,3,6$ \\
\hline Perlidae & & Marilia sp.1 & $1,2,4,7,8$ & Stenochironomus sp. & $1,4,5,7$ \\
\hline Anacroneuria $\mathrm{sp} .1$ & $1,5,7,8$ & Hydroptilidae & & Tanytarsus sp. & $1,2,3,4,5,7,9$ \\
\hline Anacroneuria sp.2 & 5 & Flintiella sp.1 & 3 & Thienemannimyia sp. & 5 \\
\hline Anacroneuria sp.3 & $2,5,7$ & Leucotrichini-gênero 1 sp.1 & 8 & Thienimanniella sp. & $1,2,3,4,5,6,7,8,9$ \\
\hline Anacroneuria jovens & 2,5 & Metrichia sp.1 & 6 & Tribelos sp. & 7 \\
\hline Hemiptera & & Hydroptila sp.1 & 1 & Chelicerata & \\
\hline Naucoridae sp. 1 & 7 & Neotrichia sp.1 & $1,3,4,5,6,7,8$ & Acari sp.1 & $1,2,3,4,5,7$ \\
\hline Coleoptera & & Leptoceridae & & ANNELIDA & \\
\hline Elmidae A sp.1 & $1,2,5,6,8$ & Oecetis sp.1 & $1,3,4,5$ & Oligochaeta & \\
\hline Elmidae A sp.2 & $5,6,7,8$ & Polycentropodidae & & Oligochaeta spp. & $1,2,3,4,5,6,7,9$ \\
\hline Noteridae A sp.1 & 1 & Polycentropus sp.1 & 6 & Hirudinea & \\
\hline Psephenidae L sp.1 & 7 & Cernotina sp.1 & 3 & Hirudinea spp. & 1,9 \\
\hline Staphylinidae A sp.1 & 8 & Lepidoptera & & MOLLUSCA & \\
\hline Staphylinidae A sp.2 & 9 & Pyralidae sp.1 & 5 & Gastropoda & \\
\hline Elmidae L sp.1 & $1,2,3,4,5,6,7,8$ & Diptera & & Gastropoda sp.1 & 9 \\
\hline Elmidae L sp.2 & $1,2,3,4,5,6,7$ & Tipulidae sp.1 & $1,2,5,7,8$ & Gastropoda sp.2 & 9 \\
\hline Elmidae L sp.3 & $1,3,4,5,6,7,8$ & Tipulidae sp.2 & 7 & Bivalvia & \\
\hline Gyrinidae L sp.1 & 9 & Tipulidae sp.3 & 9 & Bivalvia sp.1 & $3,4,9$ \\
\hline Scirtidae L sp.1 & 1 & Empididae sp.1 & $1,2,3,4,5,6,7,8$ & & \\
\hline
\end{tabular}



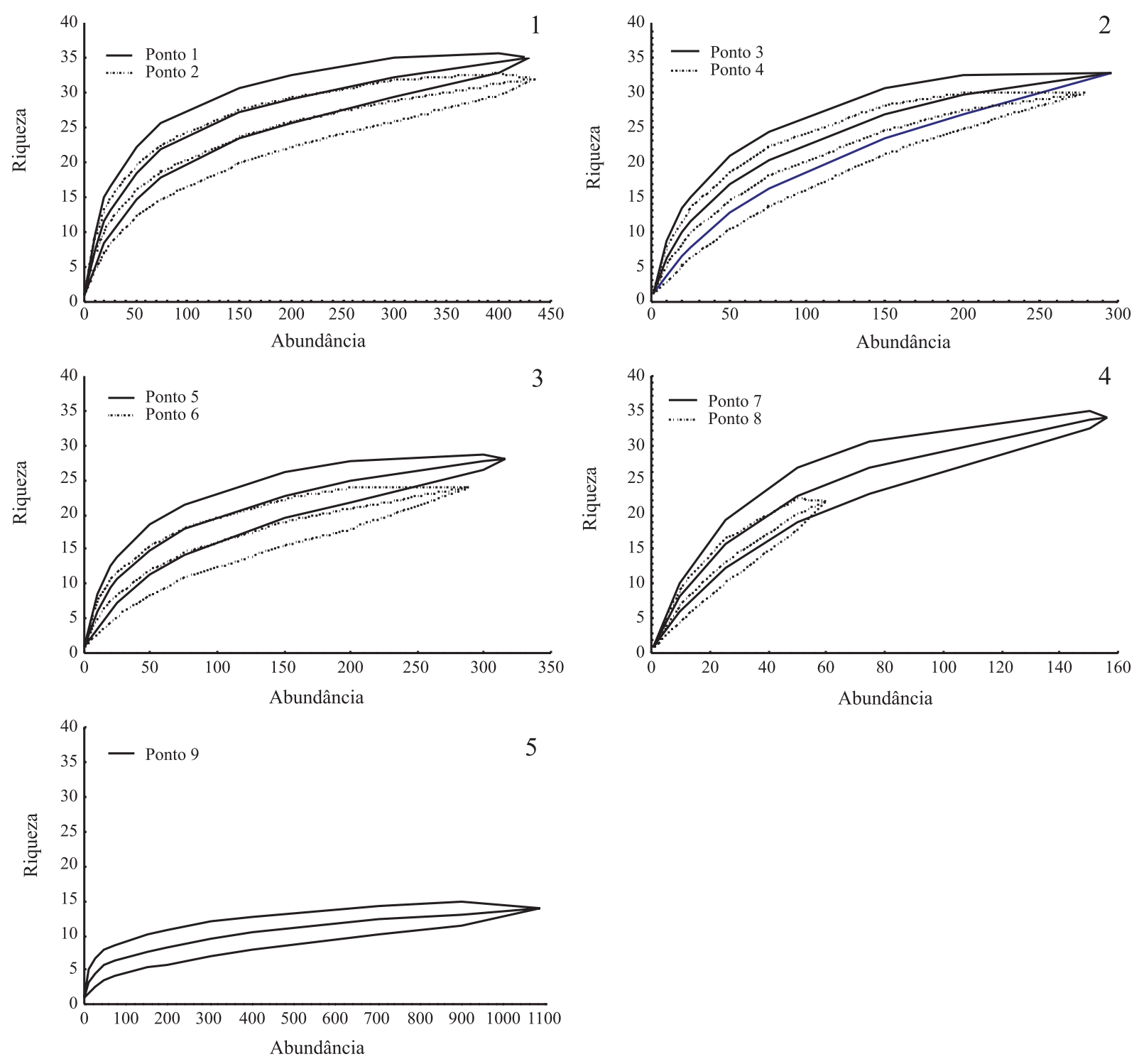

Figs. 1-5. Curvas de rarefação dos táxons referentes à fauna de macroinvertebrados aquáticos coletada em nove pontos de coleta em riachos da região de Assis, SP. Intervalo de confiança, 95\%.



Fig. 6. Riqueza padronizada para 150 indivíduos em nove pontos de coleta em riachos da região de Assis, SP. Intervalo de confiança, 95\%.

coleta. O segundo eixo explicou apenas $6,6 \%$ da variabilidade (Fig. 7). A classificação dos pontos segundo o TWINSPAN é apresentada na figura 8. A primeira divisão do TWINSPAN separou o ponto 9 (grupo

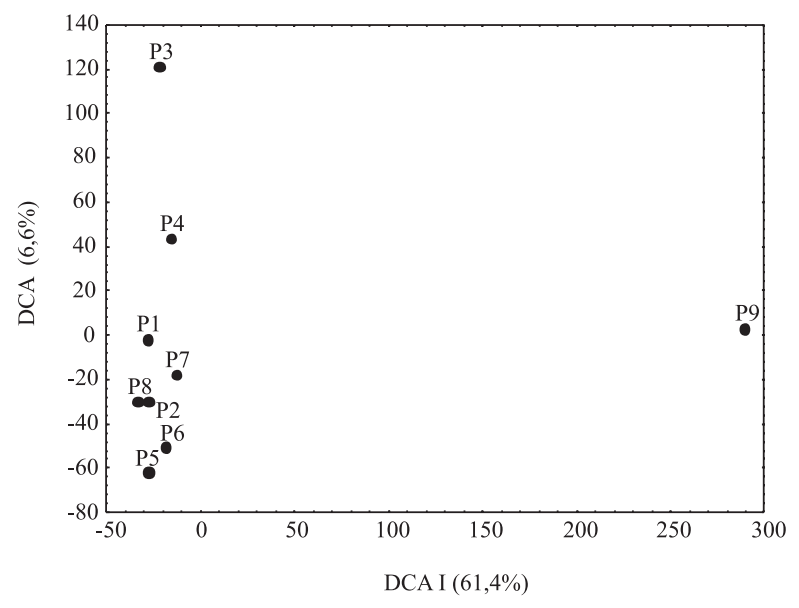

Fig. 7. Ordenação segundo a Análise de Correspondência Destendenciada (DCA) da fauna de macroinvertebrados aquáticos amostrada em nove pontos de coleta em riachos da região de Assis, Estado de São Paulo. 
D) dos demais pontos. A segunda divisão separou os pontos 2, 5 e 6 (grupo A) dos demais pontos. Já a terceira divisão separou os pontos 1, 3 e 4 (grupo B) dos pontos 7 e 8 (grupo C). O TWINSPAN mostrou que os grupos formados são determinados pelos diferentes níveis de ação antrópica observados, já que o grupo A foi formado pelos pontos com ação antrópica de intermediária a alta (intensidade 2 e 3 ), os grupos B e C com nenhuma ou baixa ação antrópica (intensidade zero e 1) e o grupo D (ponto 9), local com ação antrópica muito maior do que

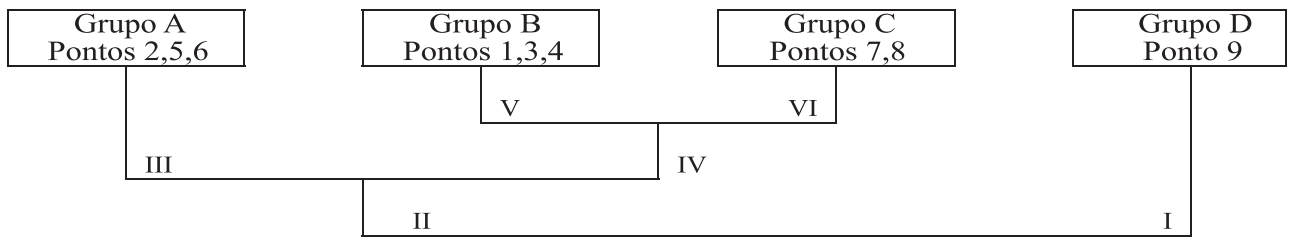

I
Colembola sp. 1 (1)
Staphylinidae A sp. 2 (1)
Gyrinidae L sp. 1 (1)
Tipulidae sp. $3(1,2)$
Chironomus sp. $(1,2,3,4,5)$
Djalmabatista sp. $(1)$
Oligochaeta spp. $(3,4,5)$
Hirudinea spp. $(1,2,3,4)$
Gastropoda sp. $1(1)$
Gastropoda sp. $2(1)$
Bivalvia sp $1(1)$

\begin{tabular}{|c|}
\hline II \\
\hline Gomphidae sp. 1 (1) \\
\hline Libellulidae sp. 1 (1) \\
\hline Libellulidae sp. $2(1,2)$ \\
\hline Libellulidae sp. 3 (1) \\
\hline Libellulidae sp. 4 (1) \\
\hline Calopterygidae sp. 1 (1) \\
\hline Anacroneuria sp. 1 (1) \\
\hline Anacroneuria sp. 3 (1) \\
\hline Elmidae L sp. $1(1,2,3)$ \\
\hline Elmidae L sp. 2 (1) \\
\hline Elmidae L sp. 3 (1) \\
\hline Leptonema sp. $1(1,2,3)$ \\
\hline Smicridea sp. 1 (1) \\
\hline Blepharopus sp. 1 (1) \\
\hline Marilia sp. 1 (1) \\
\hline Neotrichia sp 1 (1) \\
\hline Oecetis sp. 1 (1) \\
\hline Tipulidae sp. 1 (1) \\
\hline Empididae sp. 1 (1) \\
\hline Ablabesmya sp. (1) \\
\hline Beardius sp. (1) \\
\hline Tanitarsini gênero B (1) \\
\hline Corynoneura sp. (1) \\
\hline Cricotopus sp. (1) \\
\hline Lopescladius sp. (1) \\
\hline Manoa sp. (1) \\
\hline Nanocladius sp. (1) \\
\hline Parametriocnemus sp. (1) \\
\hline Pentaneura sp. (1) \\
\hline Polypedilum spp. (1) \\
\hline Pseudochironomus sp. (1) \\
\hline Rheotanytarsus sp. (2) \\
\hline Saetheria sp. (1) \\
\hline $\begin{array}{l}\text { Stenochironomus sp. (1) } \\
\text { Acari sp. } 1 \text { (1) }\end{array}$ \\
\hline
\end{tabular}

Pseudoespécies

1. 0-30 indivíduos

2. 30-60 indivíduos

3. 60-100 indivíduos

4. 100-200 indivíduos

5. $>200$ indivíduos

\begin{tabular}{|c|}
\hline III \\
\hline $\begin{array}{l}\text { Tupiara sp. } 1 \text { (1) } \\
\text { Libellulidae sp. } 3(1)\end{array}$ \\
\hline Libellulidae sp. 4 (1) \\
\hline Libellulidae sp. 5 (1) \\
\hline Anacroneuria sp. 2 (1) \\
\hline Anacroneuria sp. 3 (1) \\
\hline Elmidae L sp. $1(2,3,4)$ \\
\hline Coleoptera L sp. 2 (1) \\
\hline Coleoptera L sp. 3 (1) \\
\hline Macronema sp. 1 (1) \\
\hline Leptonema sp. $2(1,2)$ \\
\hline Leptonema $\mathrm{sp} .3$ (1) \\
\hline Smicridea sp. 1 (1) \\
\hline Smicridea sp. 2 (1) \\
\hline Metrichia sp. 1 (1) \\
\hline Polycentropus sp. 1 (1) \\
\hline Pyralidae sp. 1 (1) \\
\hline Manoa sp. (1) \\
\hline Pseudochironomus sp. (1) \\
\hline Rheotanytarsus sp. (2) \\
\hline Caladomyia sp. (2) \\
\hline Thienemannimyia sp. (1) \\
\hline
\end{tabular}

IV

Hagenulopsis sp. 1 (1)

Miroculis sp. 1 (1)

aff. Brasilocaenis sp. 1 (1)

Gomphidae sp. 2 (1)

Libellulidae sp. 1 (1)

Calopterygidae sp. 2 (1)

Naucoridae sp. 1 (1)

Noteridae A sp. 1 (1)

Psephenidae L sp. 1 (1)

Staphylinidae A sp. 1 (1)

Elmidae L sp. 2 (2)

Scirtidae L sp. 1 (1)

Coleoptera L sp. 1 (1)

Coleoptera L sp. 4 (1)

Phylloicus sp. 1 (1)

Marilia sp. 1 (1)

Flintiella sp. 1 (1)

Leucotrichini sp. 1 (1)

Hydroptila sp. 1 (1)

Cernotina sp. 1 (1)

Tipulidae sp. 2 (1)

Beardius sp. (1)

Chironomus sp. (1)

Tanitarsini gênero B (1)

Djalmabatista sp. (1)

Kiefferulus sp. (1)

Labrundinia sp. (1)

Lopescladius sp. (1)

Nanocladius sp. (1)

Parachironomus sp. (1)

Parametriocnemus sp. (1)

Phaenopsectra sp. (1)

Procladius sp. (1)

Tribelos sp. (1)

Hirudinea spp. (1)

Bivalvia sp. $1(1,2)$

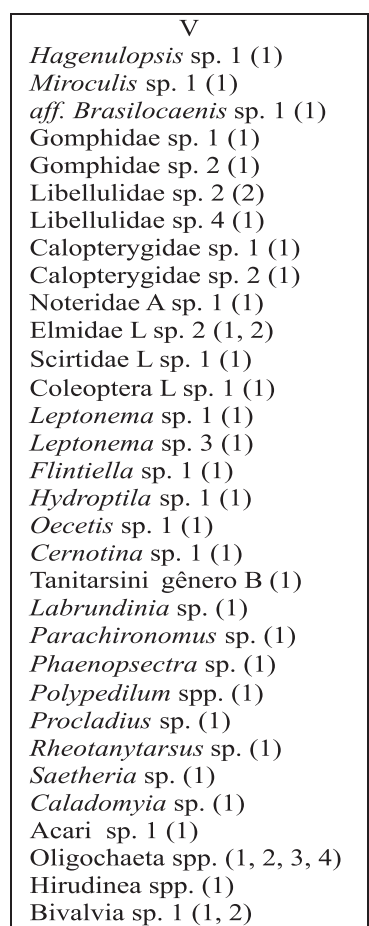

Bivalvia sp. $1(1,2)$

\section{VI}

Libellulidae sp. 3 (1)

Anacroneuria sp. 1 (1)

Anacroneuria sp. 3 (1)

Naucoridae sp. 1 (1)

Psephenidae L sp. 1 (1)

Staphylinidae A sp. 1 (1)

Coleoptera L sp. 4 (1)

Smicridea sp. 1 (1)

Blepharopus sp. 1 (1)

Phylloicus sp. 1 (1)

Leucotrichiini sp. 1 (1)

Tipulidae sp. 1 (1)

Tipulidae sp. 2 (1)

Chironomus sp. (1)

Djalmabatista sp. (1)

Fissimentum sp. (1)

Kiefferulus sp. (1)

Tribelos sp. (1)

Fig. 8. Classificação divisiva (TWINSPAN) da fauna de macroinvertebrados aquáticos amostrada em nove pontos de coleta em riachos da região de Assis, Estado de São Paulo. Nível de corte das pseudoespécies (0, 30, 60, 100 e 200). Em cada quadro são apresentadas a lista de táxons característicos de cada divisão. Os números entre parênteses se referem às classes de abundância das pseudoespécies. 
nas demais localidades (intensidade 4) (Tab. I, Fig. 8). Os táxons característicos de cada divisão, com as suas respectivas classes de abundância, também são apresentados na figura 8. Um aspecto importante em relação à primeira divisão foi a grande abundância de Chironomus sp. Meigen, 1803, Oligochaeta e Hirudinea no ponto 9 (Fig. 8). Considerando os grupos mais abundantes, foi observado que EPT (Ephemeroptera, Plecoptera e Trichoptera) e as duas espécies mais abundantes de Elmidae e Libellulidae sp. 2 variaram quanto a abundância nos diferentes pontos, porém não ocorreram no ponto 9 (Figs. 9 e 10). Por outro lado, Chironomus sp., Hirudinea e Oligochaeta aumentaram muito suas abundâncias neste ponto (Figs. 10-12).

Foi observada uma relação significativa entre a fauna e a ação antrópica (Teste de Mantel: $r=-$ $0,415, \mathrm{p}=0,007,5.000$ permutações). Por outro lado, os fatores físico-químicos não tiveram relação significativa nem com a ação antrópica (Teste de Mantel: $r=0,060, p=0,352,5.000$ permutações) nem com a fauna (Teste de Mantel: $\mathrm{r}=-0,135, \mathrm{p}=0,222$, 5.000 permutações). Não houve relação significativa entre a distância geográfica e a similaridade faunística (Teste de Mantel: r=-0,135, p=0,260, 5.000 permutações) (Tab. III).

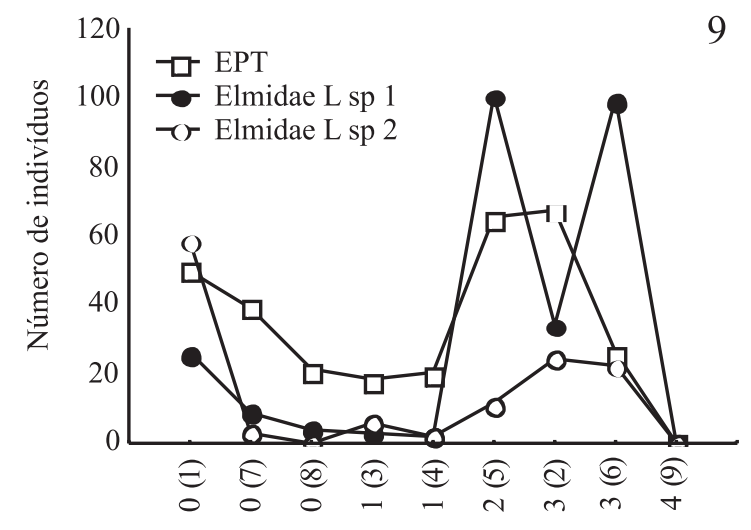

Intensidade de perturbação antrópica

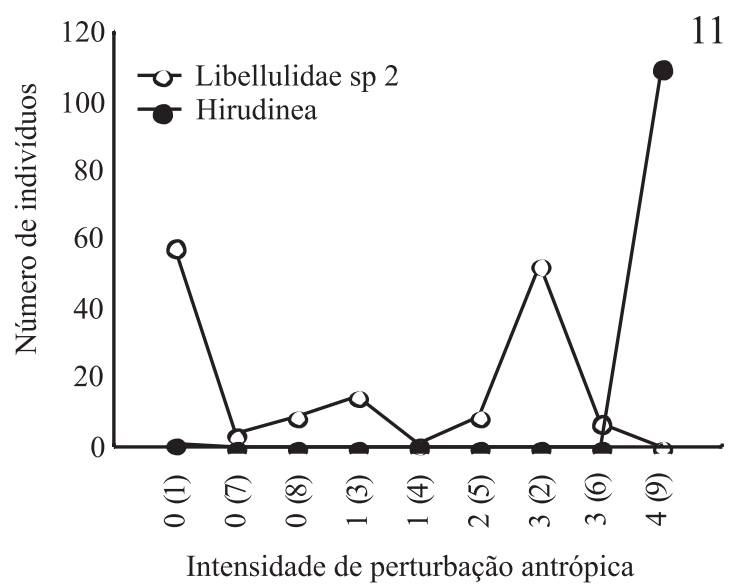

Tabela III. Teste de Mantel (5.000 permutações) relacionando o efeito dos fatores físico-químicos, da ação antrópica e da distância geográfica sobre a fauna de macroinvertebrados aquáticos e os efeitos da ação antrópica sobre os fatores físico-químicos registrados em nove pontos de coleta em riachos da região de Assis, Estado de São Paulo.

\begin{tabular}{lcc}
\hline & $\mathrm{r}$ & $\mathrm{p}$ \\
\hline Fauna & & \\
Fatores Físico-Químicos & $-0,135$ & 0,222 \\
Ação Antrópica & $-0,415$ & 0,007 \\
Distância Geográfica & $-0,135$ & 0,260 \\
\hline Ação Antrópica & & \\
Fatores Físico-Químicos & 0,060 & 0,352 \\
\hline
\end{tabular}

\section{DISCUSSÃO}

Os pontos coletados diferiram em relação à qualidade da água e à integridade estrutural (integridade do habitat e da cobertura vegetal). A vegetação não esteve intacta em nenhum dos pontos analisados. Foi observada a remoção total da vegetação ripária nos pontos 2,6 e 9 . Nos pontos 1, 4, 5 e 8, a cobertura vegetal foi parcial e apenas nos pontos 3 e 7 a vegetação cobriu a maior parte do riacho. Além da remoção da vegetação, no ponto 2 foi

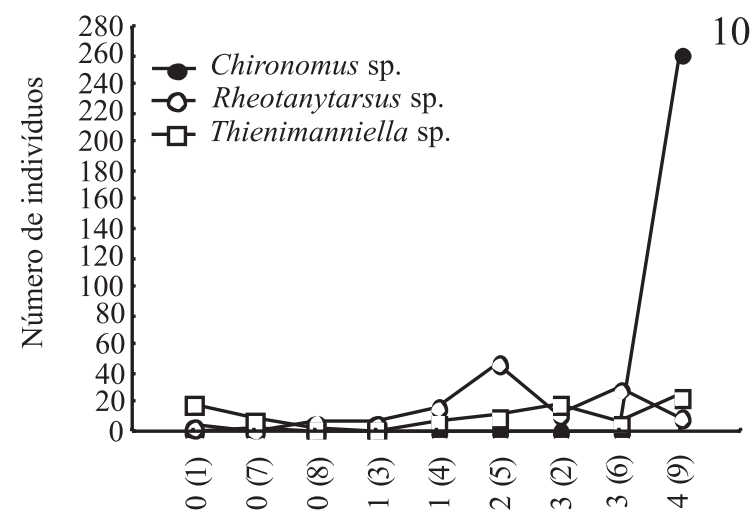

Intensidade de perturbação antrópica



Figs. 9-12. Variação da abundância dos táxons mais abundantes (n>100) em riachos da região de Assis, Estado de São Paulo. No eixo X, o primeiro valor se refere à intensidade de ação antrópica $(0$, nenhuma; 1 , baixa; 2 , média; 3, alta; 4, extremamente alta) e o segundo valor, entre parênteses, ao ponto de coleta. Ephemeroptera, Plecoptera e Trichoptera foram agrupados em EPT. 
observado um processo erosivo importante. Já o ponto 9 recebe efluentes domésticos, sendo observada uma grande poluição orgânica. Os parâmetros físico-químicos confirmam a alteração da qualidade da água no ponto 9, já que entre os locais estudados este foi o que apresentou menor $\mathrm{pH}$, maior condutividade elétrica e menor oxigênio dissolvido.

Em locais perturbados pelas atividades antrópicas há uma perda ou diminuição da importância de táxons sensíveis e o aumento da importância de táxons tolerantes, o que pode levar à diminuição da diversidade (AzRINA et al., 2006). Nesse sentido, seria esperado que a riqueza fosse negativamente relacionada com a diminuição da qualidade ambiental. O ponto 9 , local extremamente impactado pela ação antrópica, teve riqueza bem menor do que nos demais pontos. Por outro lado, alguns pontos submetidos a diferentes intensidades de ação antrópica apresentaram riquezas similares, isto indica que outros fatores devem ser importantes determinantes da riqueza nos riachos estudados. Em outras palavras, em alguns casos, os efeitos das interações bióticas e dos fatores ambientais (e.g. heterogeneidade ambiental e tamanho do riacho) podem ter sido mais importantes para a determinação da riqueza do que os efeitos depressores da ação antrópica. Por outro lado, intensidades extremas de ação antrópica, como a observada no ponto 9, devem mascarar os efeitos de todos os outros fatores sobre a riqueza, diminuindo claramente o número de táxons.

Os macroinvertebrados aquáticos utilizam diferentes faixas dentro de um gradiente ambiental e, assumindo que os efeitos locais sejam importantes, é esperado que locais com características ambientais similares tenham grupos de táxons similares. Ao contrário do esperado, os dados demonstraram que as maiores similares faunísticas não necessariamente ocorreram nos locais com fatores físico-químicos mais similares. Os resultados mostraram que a ação antrópica teve um efeito significativo sobre a fauna, mas não sobre os fatores físico-químicos. Portanto, a fauna de macroinvertebrados aquáticos e os fatores físico-químicos responderam de forma diferente aos impactos antrópicos. Tais respostas distintas podem estar relacionadas ao fato dos fatores físico-químicos refletirem o momento da coleta, o que não acontece com a fauna de macroinvertebrados aquáticos. Nesse sentido, os registros dos fatores físicoquímicos refletiriam melhor os impactos antrópicos se fossem monitorados ao longo de um determinado período de tempo, o que não aconteceu no presente trabalho. Dessa forma, as respostas dos macroinvertebrados aquáticos refletem tanto impactos crônicos quanto agudos, fornecendo uma resposta acumulativa e integradora aos efeitos de todos os impactos a que foram submetidos em um período de tempo.

Os organismos aquáticos apresentam diferentes tolerâncias às perturbações antrópicas, sendo esperado que diferentes composições faunísticas ocorram em locais com diferentes intensidades de impactos antrópicos. Esta suposição foi confirmada pelos dados do presente estudo, já que a fauna representativa dos pontos com mesma intensidade de perturbação ambiental formaram grupos. A classificação divisiva (TWINSPAN) mostrou que a fauna do ponto 9 diferiu bastante da fauna das demais localidades (primeira divisão). A segunda divisão separou os pontos 2, 5 e 6 que representam locais medianamente e altamente impactados dos pontos 1, 3 , 4, 7 e 8, locais com nenhuma ou baixa ação antrópica. No ponto 9 foram registradas altas densidades de Chironomus sp., Hirudinea e Oligochaeta, aliado a isso, foi o único local onde ocorreram espécies de Gastropoda. Com efeito, Chironomus e Oligochaeta são organismos que normalmente atingem grande abundância em locais com poluição orgânica (Goulart \& CAllisto, 2003; McCormick et al., 2004; Azrima et al., 2006). Por outro lado, alguns dos táxons mais abundantes em outros pontos não foram registrados no ponto 9 . Estes resultados demonstram os efeitos negativos dos impactos observados neste local sobre a fauna estudada.

É importante salientar que os organismos podem se dispersar entre diferentes locais, o que pode levar a uma distribuição contagiosa, ou seja, locais mais próximos podem ter faunas mais similares, fenômeno conhecido como autocorrelação espacial. Entretanto, no presente trabalho, não houve relação significativa entre a similaridade faunística e a distância geográfica. DinızFILHo et al. (1998) verificaram que o efeito da distância geográfica sobre a fauna de EPT no cerrado do Estado de Goiás foi significativo apenas na estação chuvosa (na estação seca, o efeito não foi significativo). NALLY et al. (2006), estudando o efeito da distância entre a fauna de diferentes corredeiras em dois rios australianos, verificaram que em apenas um deles a distância teve um efeito significativo sobre a fauna. Segundo aqueles autores, uma das explicações possíveis para a ausência de autocorrelação espacial em um dos rios estudados foi a ocorrência de impactos de diferentes naturezas e intensidades ao longo deste rio. Em outras palavras, os efeitos locais das diferentes condições ambientais podem diminuir a autocorrelação espacial. Neste sentido, as distintas condições observadas entre os pontos estudados, os quais diferem em relação à cobertura vegetal e à qualidade da água, poderiam explicar a ausência de autocorrelação espacial observada no presente trabalho.

O presente estudo evidenciou o efeito da qualidade ambiental sobre a fauna de macroinvertebrados aquáticos em riachos da região de Assis. A composição faunística foi influenciada pela ação antrópica, sendo que locais com mesma qualidade ambiental apresentaram maiores similaridades faunísticas. Portanto, os dados obtidos corroboram as expectativas da literatura (ROSENBERG \& Resh, 1993; Goulart \& CALlisto, 2003) e demonstram os efeitos das atividades antrópicas sobre a fauna de macroinvertebrados aquáticos.

Agradecimentos. À FAPESP (proc. 04/09711-8) pelo suporte financeiro e pela bolsa de treinamento técnico concedida a G. P. Paciencia (05/52311-3). Ao PIBIC (UNESP-reitoria e CNPq) pela bolsa de Iniciação Científica concedida a S. S. Cortezzi. Ao CNPq pela bolsa de Mestrado concedida a G. P. Paciencia. À Vera Garcia, bolsista TT-2 FAPESP, pela montagem das lâminas. Ao Ciro C. Zanini Branco e Rafael Hirata da UNESP de Assis e à Eliane Akiko Honda e Edison Adriano Berto do Instituto Florestal pelo auxílio na localização dos pontos e no trabalho de campo. 


\section{REFERÊNCIAS BIBLIOGRÁFICAS}

Azrina, M. Z.; Yap, C. K.; Ismail, A. R.; Ismael, A. \& Tan S. G. 2006. Anthropogenic impacts on the distribution and biodiversity of benthic macroinvertebrates and water quality of the Langat River, Peninsular Malaysia. Ecotoxicology and Environmental Safety 64:337-347.

Baptista, D. F.; Dorvillé, L. F. M.; Buss, D. F. \& Nessimian, J. L. 2001. Spatial and temporal organization of aquatic insect assemblages in the longitudinal gradient of a tropical river. Brazilian Journal of Biology 61(2):295-304.

Baptista, D. F.; Buss, D. F.; Egler, M.; Giovanelli, A.; Silveira, M. P. \& Nessimian, J. L. 2007. A multimetric index base on benthic macroinvertebrates for evaluation of Atlantic Forest streams at Rio de Janeiro state, Brazil. Hydrobiologia 575(1):83-94.

Barbour, M. T.; Gerritsen, J.; Griffith, G. E.; Frydenborg, R.; McCarron, E.; White, J. S. \& Bastian, M. L. 1996. A Framework for Biological Criteria for Florida Streams Using Benthic Macroinvertebrates. Journal of the North American Benthological Society 15(2):185-211.

Bispo, P. C.; Oliveira, L. G.; Bini, L. M. \& Sousa, K. G. 2006. Ephemeroptera, Plecoptera and Trichoptera assemblages from riffles in mountain streams of Central Brazil: environmental factors influencing the distribution and abundance of immatures. Brazilian Journal of Biology 66(2b):611-622.

Diniz-Filho, J. A. F.; Oliveira, L. G. \& Silva, M. M. 1998. Explaining the beta diversity of aquatic insects in "cerrado" streams from Central Brazil using multiple Mantel Test. Revista Brasileira de Biologia 58(2):223-231.

Gauch JR., H. G. 1995. Multivariate Analysis in Community Ecology. New York, Cambridge University. 298p.

Gotelli, N. J. \& Entsminger, G. L. 2000. EcoSim: Null models software for ecology. Version 5.0. Acquired Intelligence Inc. \& Kesey-Bear. Disponível em: <http://homepages. together.net/ gentsmin/ecosim.htm>. Acesso em: 2001.

Goulart, M. \& Callisto, M. 2003. Bioindicadores de qualidade de água como ferramenta em estudos de impacto ambiental. Revista FAPAM 2(2):153-164.

Hildrew, A. G. \& Townsend, C. R. 1987. Organization in Freshwater Benthic Communities. In: Gee, J. H. R. \& Giller, P. S. eds. Organization of communities: Past and Present. Oxford, Blackwell Scientific Publications. p.347-371.

Horn, H. S. 1966. Measurement of "overlap" in comparative ecological studies. American Naturalist 100(914):419-424.

Hulbert, S. H. 1971. The nonconcept of species diversity: a critique and alternative parameters. Ecology 52:577-585.

Jongman, R. H. G.; Ter BraAk, C. J. F. \& Van Tongeren, O. F. R. 1995. Data Analysis Community and Landscape Ecology. Cambridge, Cambridge University. 299p.
Krebs, C. J. 1999. Ecological Methodology. $2^{\text {nd }}$ Ed. Menlo Park, Harper \& Row. 620p.

Legendre, P. \& Legendre, L. 1998. Numerical Ecology. Developments in Environmental Modelling, 20. New York, Elsevier. $853 \mathrm{p}$.

Manly, B. F. G. 1994. A Primer of Multivariate Statistics. London, Chapmam \& Hall. 79p.

McCabe, D. J. \& Gotelli, N. G. 2000. Effects of disturbance frequency, intensity, and area on assemblages of stream macroinvertebrates. Oecologia 124:270-279.

McCormik, P. V.; Shuford, R. B. E. \& Rawlik, P. S. 2004. Changes in macroinvertebrate community structure and function along a phosphorus gradient in the Florida Everglades. Hydrobiologia 529(1-3):113-132.

McCune, B. \& Mefford, M. J. 1999. PC-ORD. Multivariate Analysis of Ecological Data. Version 4.0, MjM Software Design.

Moore, A. A. \& Palmer M. A. 2005. Invertebrate biodiversity in agricultural and urban headwater streams: implications for conservation and management. Ecological Applications 15(4): 1169-1177.

Nally, R. M.; Lloyd, N. J. \& Lake, P. S. 2006. Comparing patterns of spatial autocorrelation of assemblages of benthic invertebrates in upland rivers in south-eastern Australia. Hydrobiologia 571(1):147-156.

Rohlf, F. J. 2000. NTSYS 2.1: Numerical Taxonomic and Multivariate Analysis System. Exeter Software. 98p.

Rosenberg, D. M. \& Resh, V. H. 1993. Freshwater Biomonitoring and Benthic Macroinvertebrates. New York, Chapman \& Hall. 488p.

ShieH, S.; Kondratief, B. C. \& Ward, J. V. 1999. Longitudinal changes in benthic organic matter and macroinvertebrates in a polluted Colorado plains stream. Hydrobiologia 411(0): 191-209.

SimberlofF, D. 1972. Properties of the rarefaction diversity measurement. American Naturalist 106(949):414-418.

Vannote, R. L.; Minshall, G. W.; Cummins, K. W. L.; Sedell, J. R. \& Cushing, C. E. 1980. The River Continuum Concept. Canadian Journal of Fisheries and Aquatic Science 37:130-137.

Voeltz, N. J. \& McArthur, J. V. 2000. An exploration of factors influencing lotic insect species richness. Biodiversity Conservation 9(11): 1543-1570.

Wolda, H. 1981. Similarity indices, sample and diversity. Oecologia 50:296-302.

Zamora-MuñoZ, C. \& AlbA-Tercedor, J. 1996. Bioassessment of organically polluted Spanish rivers, using a biotic index and multivariate methods. Journal of the North American Benthological Society 15(3):332-352. 